\title{
WHO-5-Kampagne zeigt Wirkung
}

\author{
Nosokomiale Erreger werden oft durch direkten Kontakt \\ zwischen Patienten und Pflegepersonal übertragen. \\ Allerdings desinfizieren nur 38,7\% der Beschäftigten \\ konsequent ihre Hände. Eine bessere Adhärenz wäre \\ daher eine einfache und sehr effektive Maßnahme, um \\ nosokomiale Infektionen zu verhindern. \\ BM] 2015; 351: h3728
}

Die Weltgesundheitsorganisation (WHO) hat 2005 unter dem Motto „Clean care is safer care“ ein Programm ins Leben gerufen, um die Händedesinfektionsrate zu verbessern. Die Kampagne umfasst folgende 5 Maßnahmen:

- systemische Veränderungen (z.B. Verfügbarkeit von alkoholischem Händedesinfektionsmittel)
- Schulung und Weiterbildung (Bedeutung und korrekte Durchführung der Händehygiene),

- Evaluation und Feedback,

- Erinnerungen am Arbeitsplatz (z. B. in gedruckter Form, verbal oder elektronisch) und

- Etablierung einer Sicherheitskultur im Krankenhaus.
In einer Metaanalyse wurde nun untersucht, ob sich die Effekte dieses Programms messen lassen. 41 Studien erfüllten die Einschlusskriterien. 5 davon wurden in Ländern mit geringem bzw. mittlerem Einkommen durchgeführt. 10 Studien waren multizentrisch. In 19 Studien wurden zudem die Auswirkungen einer besseren Händehygiene auf die Infektionsraten untersucht. Hier zeigte sich ein Trend zu weniger MRSA-Infektionen, je mehr Desinfektionsmittel verwendet wurden. Die Rate an Clostridium-difficile-Infektionen veränderte sich nicht.

\section{Fazit}

Die WHO-5-Kampagne verbessert die Einhaltung der Händedesinfektion durch das Krankenhauspersonal. Konkrete Zielsetzungen und Anreize durch Belohnungen und Prämien könnten den Autoren zufolge den Erfolg des Programms weiter steigern. In 2 randomisierten Studien nahm dadurch die HändedesinfektionsAdhärenz nach einem Patientenkontakt um weitere $35 \% \mathrm{zu}$.

Dr. Christoph Feldmann, Köln 